\title{
COSMIC RAY SIGNATURES OF DIFFERENT TYPES OF SOLAR WIND STREAMS
}

\author{
P.K. Shrivastava* and S.P. Agrawal \\ Vikram Space Physics Centre (Physics Dept.) \\ A.P.S. University, Rewa (M.P.) 486003 \\ *Also at Govt. New Science College, \\ Rewa (M.P.) 486001
}

INTRODUCTION The earlier concept of average solar wind speed has changed with time. Besides quiet periods of low/average solar wind speeds, two different kinds of solar sources (solar flares and coronal holes) have been identified to produce high speed solar wind streams. In an earlier investigation, it was reported that the high speed streams associated to these sources produce distinctly different effects on the cosmic ray intensity (Venkatesan, et. al., 1982).

RESULT AND CONCLUSIONS The results of the chree analysis for days -5 to +8 days have been plotted, as percent deviations of the individual stations data for the two intervals of 1979-82 and 1983-86. We have used same criteria and method of analysis as adopted earlier by Venkatesan \& Co-workers. It is observed that the solar flares associated high speed solar wind streams are dominant in 1979-82, and produce much larger cosmic ray decreases at the high latitude neutron monitor stations as compared to meson monitors or low latitude neutron monitors. In contrust coronal hole associated streams are dominant during the interval of 1983-86, and produce almost equal decrease at all the stations.

REFERENCES Venkatesan, D., Shukla, A.K., and Agrawal, S.P. (1982), Cosmic ray intensity variations and two types of high speed solar streams, Solar Physics, 81, 375-81.

E. R. Priest and V. Krishan (eds.), Basic Plasma Processes on the Sun, 259-260.

(c) 1990 IAU. Printed in the Netherlands. 


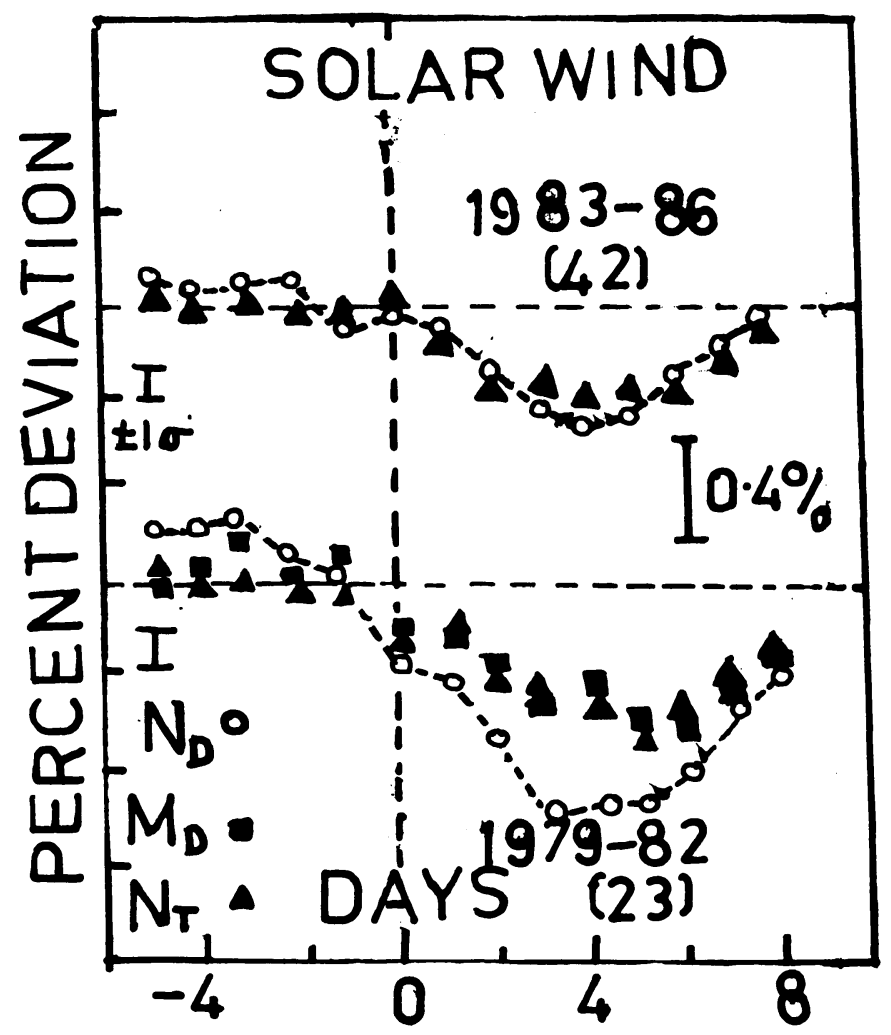

Figure 1. Shows the percent deviations of the daily mean cosmic ray intensity observed by Deep River neutron and meson monitors and those of Tokyo neutron monitor for a number of events. 